www.jmscr.igmpublication.org

Index Copernicus Value: 79.54

ISSN (e)-2347-176x ISSN (p) 2455-0450

crossref DOI: https://dx.doi.org/10.18535/jmscr/v7i6.163

\title{
A Clinical Study to Assess the QOL among Patients Undergoing Ventral Hernia Repair by Standardized Tools
}

\author{
Authors \\ Dr Thogari Kranthi Kumar, Dr T. Kiran Kumar \\ Corresponding Author \\ Dr T. Kiran Kumar
}

\begin{abstract}
The present study was done to measure Quality of life (QOL) by standardized tools and to evolve a standardized tool for measuring QOL among patients undergoing ventral hernia repair.

In our study we found that QOL as a whole improved. After 3months follow-up QOL score is better when compared to pre-operative QOL score and QOL score is even better at 6months follow-up.

Most of the subjects preferred either CCS or HerQLes.

Keywords: ventral hernia, QOL, Carolinas Comfort Scale (CCS), Short Form-36(SF-36) and Hernia Related QOL Survey (HerQLes).
\end{abstract}

\section{Introduction}

Hernia is defined as an abnormal protrusion of organ or tissue through a defect in its surrounding walls. ${ }^{1}$ Hernias of the anterior abdominal wall (ventral hernias), represent defects in the parietal abdominal wall fascia and muscle through which intra-abdominal or pre-peritoneal contents protrude. Ventral hernias may be congenital or acquired. $^{2}$

Acquired hernias may develop from slow architectural deterioration of muscular aponeuroses or from failed healing of anterior abdominal wall incision (incisional hernia). ${ }^{3}$ Incidence of umbilical hernia in adults is largely Impairment in QOL is a major reason why hernia patients seek surgical repair and changes in health-related QOL are how patients evaluate efficacy of their operations. ${ }^{7}$

This study attempts to assess QOL after ventral unknown but most cases are thought to be acquired, more common in adult females. Umbilical hernia is also commonly found in conditions of increased intra-abdominal pressure. Epigastric hernia in general population ranges from $3-5 \%$, more common in middle age, and in males $(3: 1 .)^{4}$ Incisional hernia is a common complication after abdominal surgery, incidence varying from $5-20 \% .^{5}$

Ventral hernias especially large hernias are often associated with physical, social and health problems for patient, Surgical repair remains a challenge. $^{6}$

hernia repair using CCS, SF-36, HerQLes.

\section{Materials and Methodology}

Source of data: patients undergoing ventral hernia repair at ST. ISABEL'S HOSPITAL, Chennai. 
Study period: June 2015-June 2017.

A prospective observational study.

Patients with ventral hernia and satisfying inclusion criteria

90 patients were studied.

Direct interview with patient and obtaining detailed history.

Thorough clinical examination.

Pretested structural proforma used to collect information.

\section{Inclusion criteria}

1. Patients undergoing ventral hernia repair and who are on follow-up from previous ventral hernia repair. Hernias included are umbilical, epigastric, supraumbilical and infraumbilical (paraumbilical), incisional hernias.

2. Age $>18$ years.

3. Ventral hernias in isolation or along with other hernias (ventral hernias, inguinal, femoral, lumbar, spigelian etc).

4. Patients admitted for elective ventral hernia repair.

\section{Exclusion Criteria}

1) Inguinal, femoral, obturator, parastomal \& lumbar hernias without other ventral hernias.

2) Complicated ventral hernia-with peritonitis, obstruction, strangulation.

3) Patients taken up for surgery for some other reason and incidentally found to have ventral hernia.

\section{Results}

In our study to calculate QOL using SF-36 instead of doing it in usual way, we gave rating to each question ranging from minimum of 1 to maximum of 6 depending on the variables present. 1 being best and 6 being worst. In that way we got minimum score of 36 and maximum of 148. For HerQLes, instead of rating mean score 0 to 100 , we added all the individual question score to get mean score.

QOL is divided into good, average and poor, table 1. Mean QOL in each questionnaire is calculated and used to compare the QOL pre-operatively and post-operatively.

Table 1: QOL divisions

\begin{tabular}{|l|c|c|c|}
\hline & CCS SCORE & HERQLES SCORE & SF-36 SCORE \\
\hline GOOD & $0-38$ & $12-31$ & $36-73$ \\
\hline AVERAGE & $39-76$ & $32-51$ & $74-111$ \\
\hline POOR & $77-115$ & $52-72$ & $112-148$ \\
\hline
\end{tabular}

Pre-operatively questionnaires were administered and data collected. All patients were followed up at the end of $3^{\text {rd }}$ month and $6^{\text {th }}$ month and questionnaires were re-administered.
In the following sections: pre indicates preoperative, post indicates post-operative $3^{\text {rd }}$ month, post 1 indicates post-operative $6^{\text {th }}$ month.

Age and QOL

No statistical significance in all scales, table 2.

Table 2: Age and QOL

\begin{tabular}{|c|c|c|c|c|c|c|}
\hline & \multicolumn{2}{|c|}{ Upto 50years (48\%) } & \multicolumn{2}{|c|}{$>50$ years $(52 \%)$} & \multirow{2}{*}{ P-value } \\
\hline & & Count & $\%$ & Count & $\%$ & \\
\hline \multirow{2}{*}{ CCSPRE } & GOOD & 37 & $86.0 \%$ & 41 & $87.2 \%$ & \multirow{2}{*}{0.869} \\
\hline & AVERAGE & 6 & $14.0 \%$ & 6 & $12.8 \%$ & \\
\hline CCSPOST & GOOD & 43 & $100.0 \%$ & 47 & $100.0 \%$ & --- \\
\hline \multirow{2}{*}{ CCSPOST1 } & AVERAGE & 42 & $97.7 \%$ & 47 & $100.0 \%$ & \multirow{2}{*}{0.293} \\
\hline & AVERAGE & 1 & $2.3 \%$ & 0 & $0.0 \%$ & \\
\hline \multirow{3}{*}{ HERQLESPRE } & GOOD & 6 & $14.0 \%$ & 7 & $14.9 \%$ & \multirow{3}{*}{0.931} \\
\hline & AVERAGE & 17 & $39.5 \%$ & 20 & $42.6 \%$ & \\
\hline & POOR & 20 & $46.5 \%$ & 20 & $46.5 \%$ & \\
\hline \multirow{3}{*}{ HERQLESPOST } & GOOD & 38 & $88.4 \%$ & 45 & $95.7 \%$ & \multirow{3}{*}{0.353} \\
\hline & AVERAGE & 4 & $9.3 \%$ & 2 & $4.3 \%$ & \\
\hline & POOR & 1 & $2.3 \%$ & 0 & $0.0 \%$ & \\
\hline
\end{tabular}




\begin{tabular}{|c|c|c|c|c|c|c|}
\hline \multirow{3}{*}{ HERQLESPOST1 } & GOOD & 40 & $93.0 \%$ & 47 & $100.0 \%$ & \multirow[b]{2}{*}{0.183} \\
\hline & AVERAGE & 2 & $4.7 \%$ & 0 & $0.0 \%$ & \\
\hline & POOR & 1 & $2.3 \%$ & 0 & $0.0 \%$ & \\
\hline \multirow{3}{*}{ SF-36PRE } & GOOD & 9 & $20.9 \%$ & 6 & $12.8 \%$ & \multirow{3}{*}{0.582} \\
\hline & AVERAGE & 21 & $48.8 \%$ & 25 & $53.2 \%$ & \\
\hline & POOR & 13 & $30.2 \%$ & 16 & $34.0 \%$ & \\
\hline \multirow{3}{*}{ SF-36POST } & GOOD & 12 & $27.9 \%$ & 16 & $34.0 \%$ & \multirow{3}{*}{0.418} \\
\hline & AVERAGE & 21 & $48.8 \%$ & 25 & $53.2 \%$ & \\
\hline & POOR & 10 & $23.3 \%$ & 6 & $12.3 \%$ & \\
\hline \multirow{3}{*}{ SF-36POST1 } & GOOD & 32 & $74.4 \%$ & 37 & $78.7 \%$ & \multirow{3}{*}{0.552} \\
\hline & AVERAGE & 10 & $23.3 \%$ & 10 & $21.3 \%$ & \\
\hline & POOR & 1 & $2.3 \%$ & 0 & $0.0 \%$ & \\
\hline
\end{tabular}

\section{Gender and QOL}

No statistical significance in all scales, table 3.

Table 3: Gender and QOL

\begin{tabular}{|c|c|c|c|c|c|c|}
\hline & \multicolumn{2}{|c|}{ Female(74\%) } & \multicolumn{2}{|c|}{ Male(26\%) } & \multirow{2}{*}{ P-value } \\
\hline & & Count & $\%$ & Count & $\%$ & \\
\hline \multirow{2}{*}{ CCSPRE } & GOOD & 59 & $88.1 \%$ & 19 & $82.6 \%$ & \multirow{2}{*}{0.507} \\
\hline & AVERAGE & 8 & $1.9 \%$ & 4 & $17.4 \%$ & \\
\hline CCSPOST & GOOD & 67 & $100.0 \%$ & 23 & $100.0 \%$ & --- \\
\hline \multirow{2}{*}{ CCSPOST1 } & GOOD & 66 & $98.5 \%$ & 23 & $100.0 \%$ & \multirow{2}{*}{0.556} \\
\hline & AVERAGE & 1 & $1.5 \%$ & 0 & $0.0 \%$ & \\
\hline \multirow{3}{*}{ HERQLESPRE } & GOOD & 8 & $11.9 \%$ & 5 & $21.7 \%$ & \multirow{3}{*}{0.242} \\
\hline & AVERAGE & 26 & $38.8 \%$ & 11 & $47.8 \%$ & \\
\hline & POOR & 33 & $49.3 \%$ & 7 & $30.4 \%$ & \\
\hline \multirow{3}{*}{$\begin{array}{l}\text { HERQLESPOS } \\
T\end{array}$} & GOOD & 61 & $91.0 \%$ & 22 & $95.7 \%$ & \multirow{3}{*}{0.729} \\
\hline & AVERAGE & 5 & $7.5 \%$ & 1 & $4.3 \%$ & \\
\hline & POOR & 1 & $1.5 \%$ & 0 & $0.0 \%$ & \\
\hline \multirow{3}{*}{$\begin{array}{l}\text { HERQLESPOS } \\
\text { T1 }\end{array}$} & GOOD & 64 & $95.5 \%$ & 23 & $100.0 \%$ & \multirow{3}{*}{0.587} \\
\hline & AVERAGE & 2 & $3.0 \%$ & 0 & $0.0 \%$ & \\
\hline & POOR & 1 & $1.5 \%$ & 0 & $0.0 \%$ & \\
\hline \multirow{3}{*}{ SF-36PRE } & GOOD & 12 & $17.9 \%$ & 3 & $13.0 \%$ & \multirow{3}{*}{0.855} \\
\hline & AVERAGE & 34 & $50.7 \%$ & 12 & $52.2 \%$ & \\
\hline & POOR & 21 & $31.3 \%$ & 3 & $34.8 \%$ & \\
\hline \multirow{3}{*}{ SF-36POST } & GOOD & 20 & $29.9 \%$ & 8 & $34.8 \%$ & \multirow{3}{*}{0.146} \\
\hline & AVERAGE & 32 & $47.8 \%$ & 14 & $60.9 \%$ & \\
\hline & POOR & 15 & $22.4 \%$ & 1 & $4.3 \%$ & \\
\hline \multirow{3}{*}{ SF-36POST1 } & GOOD & 49 & $73.1 \%$ & 20 & $87.0 \%$ & \multirow{3}{*}{0.379} \\
\hline & AVERAGE & 17 & $25.4 \%$ & 3 & $13.0 \%$ & \\
\hline & POOR & 1 & $1.5 \%$ & 0 & $0.0 \%$ & \\
\hline
\end{tabular}

\section{BMI and QOL}

No statistical significance in all scales, table 4.

Table 4: BMI and QOL

\begin{tabular}{|c|c|c|c|c|c|c|c|c|}
\hline & & \multicolumn{2}{|c|}{ NORMAL(19\%) } & \multicolumn{2}{|c|}{ OVERWEIGHT(42\%) } & \multicolumn{2}{|c|}{ OBESE(39\%) } & \multirow{2}{*}{ P-value } \\
\hline & & Count & $\%$ & Count & $\%$ & Count & $\%$ & \\
\hline \multirow{2}{*}{ CCSPRE } & GOOD & 14 & $82.4 \%$ & 31 & $81.6 \%$ & 33 & $94.3 \%$ & \multirow{2}{*}{0.237} \\
\hline & AVERAGE & 3 & $17.6 \%$ & 7 & $18.4 \%$ & 2 & $5.7 \%$ & \\
\hline CCSPOST & GOOD & 17 & $100.0 \%$ & 38 & $100.0 \%$ & 35 & $100.0 \%$ & --- \\
\hline \multirow{2}{*}{ CCSPOST1 } & GOOD & 16 & $94.1 \%$ & 38 & $100.0 \%$ & 35 & $100.0 \%$ & \multirow{2}{*}{0.114} \\
\hline & AVERAGE & 1 & $5.9 \%$ & 0 & $0.0 \%$ & 0 & $0.0 \%$ & \\
\hline \multirow{3}{*}{ HERQLESPRE } & GOOD & 2 & $11.8 \%$ & 5 & $13.2 \%$ & 6 & $17.1 \%$ & \multirow{3}{*}{0.931} \\
\hline & AVERAGE & 10 & $58.8 \%$ & 13 & $34.2 \%$ & 14 & $40.0 \%$ & \\
\hline & POOR & 5 & $29.4 \%$ & 20 & $52.6 \%$ & 15 & $42.9 \%$ & \\
\hline \multirow[b]{2}{*}{ HERQLESPOST } & GOOD & 14 & $82.4 \%$ & 37 & $97.4 \%$ & 32 & $91.4 \%$ & \multirow{2}{*}{0.173} \\
\hline & AVERAGE & 2 & $11.8 \%$ & 1 & $2.6 \%$ & 3 & $8.6 \%$ & \\
\hline
\end{tabular}




\begin{tabular}{|c|c|c|c|c|c|c|c|c|}
\hline & POOR & 1 & $5.9 \%$ & 0 & $0.0 \%$ & 0 & $0.0 \%$ & \\
\hline \multirow{3}{*}{ HERQLESPOST1 } & GOOD & 16 & $94.1 \%$ & 38 & $100.0 \%$ & 33 & $94.3 \%$ & \multirow{3}{*}{0.111} \\
\hline & AVERAGE & 0 & $0.0 \%$ & 0 & $0.0 \%$ & 2 & $5.7 \%$ & \\
\hline & POOR & 1 & $5.9 \%$ & 0 & $0.0 \%$ & 0 & $0.0 \%$ & \\
\hline \multirow{3}{*}{ SF-36PRE } & GOOD & 2 & $11.8 \%$ & 6 & $15.8 \%$ & 7 & $20.0 \%$ & \multirow{3}{*}{0.888} \\
\hline & AVERAGE & 8 & $47.1 \%$ & 20 & $52.6 \%$ & 18 & $51.4 \%$ & \\
\hline & POOR & 7 & $41.2 \%$ & 12 & $31.6 \%$ & 10 & $28.6 \%$ & \\
\hline \multirow{3}{*}{ SF-36POST } & GOOD & 5 & $29.4 \%$ & 10 & $26.3 \%$ & 13 & $37.1 \%$ & \multirow{3}{*}{0.789} \\
\hline & AVERAGE & 8 & $47.1 \%$ & 22 & $57.9 \%$ & 16 & $45.7 \%$ & \\
\hline & POOR & 4 & $23.5 \%$ & 6 & $15.8 \%$ & 6 & $17.1 \%$ & \\
\hline \multirow{3}{*}{ SF-36POST1 } & GOOD & 12 & $70.6 \%$ & 32 & $84.2 \%$ & 25 & $71.4 \%$ & \multirow{3}{*}{0.189} \\
\hline & AVERAGE & 4 & $23.5 \%$ & 6 & $15.8 \%$ & 10 & $28.6 \%$ & \\
\hline & POOR & 1 & $5.9 \%$ & 0 & $0.0 \%$ & 0 & $0.0 \%$ & \\
\hline
\end{tabular}

\section{Defect Size and QOL}

Statistically significant difference in QOL pre-operatively in SF-36, table 5.

Table 5: Defect size and QOL

\begin{tabular}{|c|c|c|c|c|c|c|}
\hline & \multicolumn{2}{|c|}{$<=3 \operatorname{CMS}(67 \%)$} & \multicolumn{2}{|c|}{$>3 \mathrm{CMS}(33 \%)$} & \multirow{2}{*}{ P-value } \\
\hline & & Count & $\%$ & Count & $\%$ & \\
\hline \multirow{2}{*}{ CCSPRE } & GOOD & 50 & $83.3 \%$ & 28 & $93.3 \%$ & \multirow{2}{*}{0.188} \\
\hline & AVERAGE & 10 & $16.7 \%$ & 2 & $6.7 \%$ & \\
\hline CCSPOST & GOOD & 60 & $100.0 \%$ & 30 & $100.0 \%$ & --- \\
\hline \multirow{2}{*}{ CCSPOST1 } & GOOD & 59 & $98.3 \%$ & 30 & $100.0 \%$ & \multirow{2}{*}{0.477} \\
\hline & AVERAGE & 1 & $1.7 \%$ & 0 & $0.0 \%$ & \\
\hline \multirow{3}{*}{ HERQLESPRE } & GOOD & 8 & $13.3 \%$ & 5 & $16.7 \%$ & \multirow{3}{*}{0.914} \\
\hline & AVERAGE & 25 & $41.7 \%$ & 12 & $40.0 \%$ & \\
\hline & POOR & 27 & $45.0 \%$ & 13 & $14.4 \%$ & \\
\hline \multirow{3}{*}{$\begin{array}{l}\text { HERQLESPOS } \\
\text { T }\end{array}$} & GOOD & 54 & $90.0 \%$ & 29 & $96.7 \%$ & \multirow{3}{*}{0.510} \\
\hline & AVERAGE & 5 & $8.3 \%$ & 1 & $3.3 \%$ & \\
\hline & POOR & 1 & $1.7 \%$ & 0 & $0.0 \%$ & \\
\hline \multirow{3}{*}{$\begin{array}{l}\text { HERQLESPOS } \\
\text { T1 }\end{array}$} & GOOD & 58 & $96.7 \%$ & 29 & $96.7 \%$ & \multirow{3}{*}{0.687} \\
\hline & AVERAGE & 1 & $1.7 \%$ & 1 & $1.3 \%$ & \\
\hline & POOR & 1 & $1.7 \%$ & 0 & $0.0 \%$ & \\
\hline \multirow{3}{*}{ SF-36PRE } & GOOD & 12 & $20.0 \%$ & 3 & $10.0 \%$ & \multirow{3}{*}{0.040} \\
\hline & AVERAGE & 25 & $41.7 \%$ & 21 & $70.0 \%$ & \\
\hline & POOR & 23 & $38.3 \%$ & 6 & $20.0 \%$ & \\
\hline \multirow{3}{*}{ SF-36POST } & GOOD & 21 & $35.0 \%$ & 7 & $23.3 \%$ & \multirow{3}{*}{0.261} \\
\hline & AVERAGE & 27 & $45.0 \%$ & 19 & $63.3 \%$ & \\
\hline & POOR & 12 & $20.0 \%$ & 4 & $13.3 \%$ & \\
\hline \multirow{3}{*}{ SF-36POST1 } & GOOD & 44 & $73.3 \%$ & 25 & $83.3 \%$ & \multirow{3}{*}{0.500} \\
\hline & AVERAGE & 15 & $25.0 \%$ & 5 & $16.7 \%$ & \\
\hline & POOR & 1 & $1.7 \%$ & 0 & $0.0 \%$ & \\
\hline
\end{tabular}

\section{Surgery}

Data regarding ventral hernia repair only shown. (Other surgeries like inguinal hernia, hysterectomy etc. not shown).

Table 6: Type of surgery and QOL

\begin{tabular}{|c|c|c|c|c|c|c|}
\hline & \multicolumn{2}{|c|}{ LAPAROSCOPY $(5.6 \%)$} & \multicolumn{2}{|c|}{ OPEN(94.4\%) } & \multirow{2}{*}{ P-value } \\
\hline & & Count & $\%$ & Count & $\%$ & \\
\hline \multirow{2}{*}{ CCSPRE } & GOOD & 5 & $100.0 \%$ & 73 & $85.9 \%$ & \multirow{2}{*}{0.367} \\
\hline & AVERAGE & 0 & $0.0 \%$ & 12 & $14.1 \%$ & \\
\hline CCSPOST & GOOD & 5 & $100.0 \%$ & 85 & $100.0 \%$ & --- \\
\hline \multirow{2}{*}{ CCSPOST1 } & GOOD & 5 & $100.0 \%$ & 84 & $98.8 \%$ & \multirow{2}{*}{0.807} \\
\hline & AVERAGE & 0 & $0.0 \%$ & 1 & $1.2 \%$ & \\
\hline HERQLESPRE & GOOD & 1 & $20.0 \%$ & 12 & $14.1 \%$ & 0.933 \\
\hline
\end{tabular}




\begin{tabular}{|c|c|c|c|c|c|c|}
\hline & AVERAGE & 2 & $40.0 \%$ & 35 & $41.2 \%$ & \\
\hline & POOR & 2 & $40.0 \%$ & 38 & $44.7 \%$ & \\
\hline \multirow{3}{*}{$\begin{array}{l}\text { HERQLESPOS } \\
T\end{array}$} & GOOD & 5 & $100.0 \%$ & 78 & $91.8 \%$ & \multirow{3}{*}{0.800} \\
\hline & AVERAGE & 0 & $0.0 \%$ & 6 & $7.1 \%$ & \\
\hline & POOR & 0 & $0.0 \%$ & 1 & $1.2 \%$ & \\
\hline \multirow{3}{*}{$\begin{array}{l}\text { HERQLESPOS } \\
\text { T1 }\end{array}$} & GOOD & 5 & $100.0 \%$ & 82 & $96.5 \%$ & \multirow{3}{*}{0.913} \\
\hline & AVERAGE & 0 & $0.0 \%$ & 2 & $2.4 \%$ & \\
\hline & POOR & 0 & $0.0 \%$ & 1 & $1.2 \%$ & \\
\hline \multirow{3}{*}{ SF-36PRE } & GOOD & 3 & $60.0 \%$ & 12 & $14.1 \%$ & \multirow{3}{*}{0.027} \\
\hline & AVERAGE & 1 & $20.0 \%$ & 45 & $52.9 \%$ & \\
\hline & POOR & 1 & $20.0 \%$ & 28 & $32.9 \%$ & \\
\hline \multirow{3}{*}{ SF-36POST } & GOOD & 3 & $60.0 \%$ & 25 & $29.4 \%$ & \multirow{3}{*}{0.288} \\
\hline & AVERAGE & 2 & $40.0 \%$ & 44 & $51.8 \%$ & \\
\hline & POOR & 0 & $0.0 \%$ & 16 & $18.8 \%$ & \\
\hline \multirow{3}{*}{ SF-36POST1 } & GOOD & 5 & $100.0 \%$ & 64 & $75.3 \%$ & \multirow{3}{*}{0.447} \\
\hline & AVERAGE & 0 & $0.0 \%$ & 20 & $23.5 \%$ & \\
\hline & POOR & 0 & $0.0 \%$ & 1 & $1.2 \%$ & \\
\hline
\end{tabular}

\section{Type of Repair and QOL}

Statistically significant difference in QOL post-operatively $3^{\text {rd }}$ month using HerQLes survey, table 7.

Table 7: Type of repair and QOL

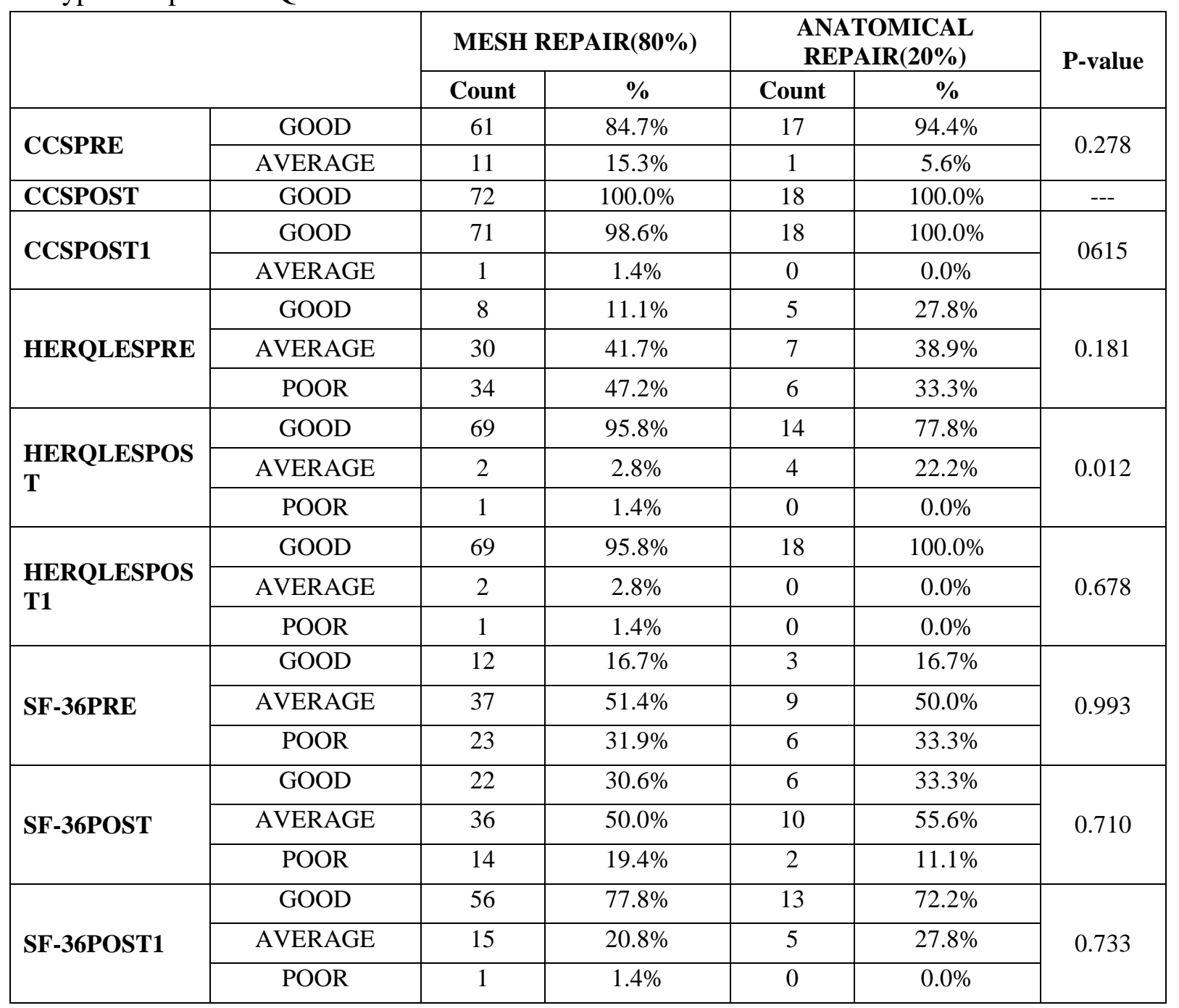


Mesh Placement and QOL

No statistical significant difference, table 8 .

Table 8: Mesh Placement and QOL

\begin{tabular}{|c|c|c|c|c|c|c|c|c|}
\hline & & \multicolumn{2}{|c|}{ ONLAY $(74 \%)$} & \multicolumn{2}{|c|}{ INTRAPERITONEAL $(6 \%)$} & \multicolumn{2}{|c|}{$\mathrm{NA}(20 \%)$} & \multirow{2}{*}{ P-value } \\
\hline & & Count & $\%$ & Count & $\%$ & Count & $\%$ & \\
\hline \multirow{2}{*}{ CCSPRE } & GOOD & 56 & $83.6 \%$ & 5 & $100.0 \%$ & 17 & $94.4 \%$ & \multirow{2}{*}{0.323} \\
\hline & AVERAGE & 11 & $16.4 \%$ & 0 & $0.0 \%$ & 1 & $5.6 \%$ & \\
\hline CCSPOST & GOOD & 67 & $100.0 \%$ & 5 & $100.0 \%$ & 18 & $100.0 \%$ & -- \\
\hline \multirow{2}{*}{ CCSPOST1 } & GOOD & 66 & $98.5 \%$ & 5 & $100.0 \%$ & 18 & $100.0 \%$ & \multirow{2}{*}{0.841} \\
\hline & AVERAGE & 1 & $1.5 \%$ & 0 & $0.0 \%$ & 0 & $0.0 \%$ & \\
\hline \multirow{3}{*}{ HERQLESPRE } & GOOD & 7 & $10.4 \%$ & 1 & $20.0 \%$ & 5 & $27.8 \%$ & \multirow{3}{*}{0.436} \\
\hline & AVERAGE & 28 & $41.8 \%$ & 2 & $40.0 \%$ & 7 & $38.9 \%$ & \\
\hline & POOR & 32 & $47.8 \%$ & 2 & $40.0 \%$ & 6 & $33.3 \%$ & \\
\hline \multirow{3}{*}{ HERQLESPOST } & GOOD & 64 & $95.5 \%$ & 5 & $100.0 \%$ & 14 & $77.8 \%$ & \multirow{3}{*}{0.059} \\
\hline & AVERAGE & 2 & $3.0 \%$ & 0 & $0.0 \%$ & 4 & $22.2 \%$ & \\
\hline & POOR & 1 & $1.5 \%$ & 0 & $0.0 \%$ & 0 & $0.0 \%$ & \\
\hline \multirow{3}{*}{ HERQLESPOST1 } & GOOD & 64 & $95.5 \%$ & 5 & $100.0 \%$ & 18 & $100.0 \%$ & \multirow{3}{*}{0.900} \\
\hline & AVERAGE & 2 & $3.0 \%$ & 0 & $0.0 \%$ & 0 & $0.0 \%$ & \\
\hline & POOR & 1 & $1.5 \%$ & 0 & $0.0 \%$ & 0 & $0.0 \%$ & \\
\hline \multirow{3}{*}{ SF-36PRE } & GOOD & 9 & $13.4 \%$ & 3 & $60.0 \%$ & 3 & $16.7 \%$ & \multirow{3}{*}{0.119} \\
\hline & AVERAGE & 36 & $53.7 \%$ & 1 & $20.0 \%$ & 9 & $50.0 \%$ & \\
\hline & POOR & 22 & $32.8 \%$ & 1 & $20.0 \%$ & 6 & $33.3 \%$ & \\
\hline \multirow{3}{*}{ SF-36POST } & GOOD & 19 & $28.4 \%$ & 3 & $60.0 \%$ & 6 & $33.3 \%$ & \multirow{3}{*}{0.489} \\
\hline & AVERAGE & 34 & $50.7 \%$ & 2 & $40.0 \%$ & 10 & $55.6 \%$ & \\
\hline & POOR & 14 & $20.9 \%$ & 0 & $0.0 \%$ & 2 & $11.1 \%$ & \\
\hline \multirow{3}{*}{ SF-36POST1 } & GOOD & 51 & $76.1 \%$ & 5 & $100.0 \%$ & 13 & $72.2 \%$ & \multirow{3}{*}{0.716} \\
\hline & AVERAGE & 15 & $22.4 \%$ & 0 & $0.0 \%$ & 5 & $27.8 \%$ & \\
\hline & POOR & 1 & $1.5 \%$ & 0 & $0.0 \%$ & 0 & $0.0 \%$ & \\
\hline
\end{tabular}

\section{QOL}

\section{Pre-operative:}

CCS: Mean score-24.03, statistically significant $(\mathrm{p}=0.000)$. 78(86.7\%) had good and 12(13.3\%) had average QOL.

HERQLES: Mean score-46.78, statistically significant $\quad(\mathrm{p}=0.000) .13(14.4 \%)$ had good, $37(41.1 \%)$ had average and $40(44.4 \%)$ had poor QOL.

SF-36: Mean score-98.49, statistically significant $(\mathrm{p}=0.000) .15(16.7 \%)$ had good, 46(51.1\%) had average and 29(32.2\%) had poor QOL.

\section{Post-operative $3^{\text {rd }}$ month:}

CCS: Mean score-6.08, statistically significant $(p=0.000)$.All $(100 \%)$ had good QOL.

HERQLES: Mean score-18.72, statistically significant $(\mathrm{p}=0.000) .83(92.2 \%)$ had good, $6(6.7 \%)$ had average and $1(1.1 \%)$ had poor QOL.

SF-36: Mean score-89.63, statistically significant $(\mathrm{p}=0.000) .28(31.1 \%)$ had good, 46(51.1\%) had average and $16(17.8 \%)$ had poor QOL.

\section{Post-operative $6^{\text {th }}$ month:}

CCS: Mean score-3.89, statistically significant $(\mathrm{p}=0.000) .89(98.9 \%)$ had good and $1(1.1 \%)$ had average QOL.

HERQLES: Mean score-15.76, statistically significant $(\mathrm{p}=0.000) .87(96.7 \%)$ had good, $2(2.2 \%)$ had average and $1(1.1 \%)$ had poor QOL.

SF-36: Mean score-63.23, statistically significant $(\mathrm{p}=0.000)$. 69(76.7\%) had good, 20(22.2\%) had average and $1(1.1 \%)$ had poor QOL.

\section{QOL Scores Comparison}

Pre-Operative and $3^{\text {rd }}$ Month Post-Operative Mean Scores

CCS: Mean difference-17.956, statistically significant ( $p=0.000)$.

HERQLES: Mean difference-28.056, statistically significant ( $\mathrm{p}=0.000)$.

SF-36: Mean difference-8.856, statistically significant $(\mathrm{p}=0.002)$. 
Pre-Operative and $6^{\text {th }}$ month Post-Operative Mean Scores

CCS: Mean difference-20.144, statistically significant $(\mathrm{p}=0.000)$.

HERQLES: Mean difference-31.022, statistically significant $(\mathrm{p}=0.000)$.

SF-36: Mean difference-35.256, statistically significant $(\mathrm{p}=0.000)$.

$3^{\text {rd }}$ month and $6^{\text {th }}$ month Post-Operative Mean Scores

CCS: Mean difference-2.189, statistically significant $(\mathrm{p}=0.000)$.

HERQLES: M ean difference-2.967, statistically significant $(\mathrm{p}=0.000)$.

SF-36:Mean difference-26.400, statistically significant $(\mathrm{p}=0.000)$.

Among the three questionnaires, 44(48.89\%) preferred HerQLes, 41(45.56\%) preferred CCS, $5(5.55 \%)$ preferred SF-36.

\section{Discussion}

Our study has subjects ranging from age 3185 year (mean age 52.6years). We divided subjects into two groups as age $<=50$ years and $>50$ years. $43(47.8 \%)$ are age $<=50$ years and $47(52.2 \%)$ are $>50$ years. QOL in comparison to age showed no statistical significance.

Ladurner R, Chiapponi C, Linhuber Q, et al ${ }^{8}$ found no significant difference in SF-36(QOL) with age after open incisional hernia repair with light or heavy weight mesh (p-value 0.840). In our study even though we did not compare QOL in relation to mesh against age, SF-36 scores did not show any significant difference in QOL with age after 6 months (p-value 0.552).

Our study has 67 females (74.4\%) and 23 males (25.6\%). We found gender has no effect on QOL.

Average BMI in our study is 29.03. We divided our subjects into 3 groups based on BMI. Normal weight up to 24.99 , overweight 25-29.99, and obese $>=30$. Normal weight are $17(18.9 \%)$, overweight are $38(42.2 \%)$, and $35(38.9 \%)$ are obese. When comparing QOL with BMI we found no statistical significance.
Krpata DM, Schmotzer BJ, Flocke S,et al $^{9}$ using HerQLes, found no difference in QOL after with age (p-value 0.21), gender(p- value 0.88) and $\mathrm{BMI}(\mathrm{p}$-value 0.21). Our study also did not find any difference in QOL after 6months in HerQLes (p-value 0.183).

Though clinical examination and radiological investigations were used, defect size found intraoperatively was taken as final. We divided subjects into 2 groups based on defect size. One group in which defect was taken as $=<3 \mathrm{cms}$ constituted $66.7 \%$ (60), other group in which defect was $>3 \mathrm{cms}$ constituted $33.3 \%$ (30)(Average defect size is $3.144 \mathrm{cms}$ ). We found statistically significant difference in QOL pre-operatively in SF-36 scale (p-value0.040), but there was no difference post-operatively using SF-36, and CCS, HerQLes.

Ladurner R, Chiapponi C, Linhuber Q, et al ${ }^{8}$ using SF-36 scale found that QOL with defect size was not significant (p-value0.292). Our study also showed similar results ( $\mathrm{p}$-value 0.500 ).

Of 90subjects, $38(42.2 \%)$ had umbilical hernia, $2(2.2 \%)$ epigastric hernia, 10(11.1\%) paraumbilical hernia, 27(30\%) incisional hernia, $5(5.6 \%)$ recurrent incisional hernia, 8(8.9\%) combined ventral hernia. Diagnosis like inguinal hernia, cholelithiasis etc. are not considered.

$85(94.4 \%)$ underwent open and 5(5.6\%) laparoscopic repair. There was statistically significant difference in QOL pre-operatively using SF-36 scale (p-value 0.027), but not postoperatively, also no statistical significant difference in QOL in CCS, HerQLes.

Hope WW, Lincourt AE, Newcomb WL,et $\mathrm{al}^{10}$ found no difference in preoperative QOL scores in SF-36 between laparoscopic/open repair. Postoperative QOL scores in SF-36 and CCS were significantly improved in laparoscopic group. They had 41(73\%) laparoscopic and 15(27\%) open repairs. Our study had more open than laparoscopic repairs, the difference in results may be attributed to variability in percentage of patients undergoing laparoscopic and open repair. 
Colavita PD, Tsirline VB, Belyansky I,et al ${ }^{11}$ using CCS found no difference in QOL after laparoscopic/open ventral hernia repair at 6 follow-up.

$18(20 \%)$ underwent anatomical repair, 72(80\%) underwent mesh repair. In our study, there was statistically significant difference in QOLat 3 months follow-up using HerQLes, but not preoperatively or post-operative 6 months. There was no statistical significant difference in QOL in CCS, SF-36 scales.

Bard mesh was used in 13(14.4\%), parietex mesh in $12(13.3 \%)$, polyester mesh in $1(1.1 \%)$, prolene mesh in $36(40 \%)$, soft prolene mesh in $5(5.6 \%)$, composite polypropylene mesh in $1(1.1 \%)$, provisc 3D mesh in 4(4.4\%). Mesh was placed as Onlay in $67(74.4 \%)$ and as intraperitoneal mesh in $5(5.6 \%)$. We did not find statistically significant difference in QOL with mesh placement.

Ladurner R, Chiapponi C, Linhuber Q, et $\mathrm{al}^{8}$ comparing QOL using SF-36 after open incisional hernia repair with light versus heavy weight meshes found that QOL was not related to mesh type.

There is a significant improvement in postoperative QOL mean scores at 3months and 6 months in all scales when compared to preoperative mean scores. There is also increase in number of subjects having good and average QOL at the end of study.

Mette W. Christoffersen, et $\mathrm{al}^{12}$ demonstrated significant changes in QOL with CCS after 90days. Our study also showed improvement in QOL.

David A Klima,et al ${ }^{13}$ noted mean CCS score of 2.18 after open repair at 6-12 months which is comparable to our study.

Krpata DM, Schmotzer BJ, Flocke $S$, et $\mathrm{al}^{9}$ showed significant improvement in QOL using HerQLes after 6months(P-value <0.001). Our study also had improved QOL after 6 months (p-value 0.000).

Mussack T, Ladurner R, Vogel T, et al ${ }^{15}$ showed significant improvement in QOL scores after 28 months with SF-36(open repair). Our study also showed improvement in QOL after 6months.

Most of the subjects preferred either CCS $(41.56 \%)$ or HerQLes (48.59\%) over SF$36(5.55 \%)$. Because most of the subjects felt that CCS or HerQLes are addressing their disease specific QOL in satisfactory way, either CCS or HerQLes can be used in our Indian setting with replacing movement limitation in CCS with "abdominal wall interference" or removing my abdominal wall interferes with my sexual activity, I often feel blue because of my abdominal wall in HerQLes. OR If I was given a chance to validate a tool in the Indian setting, based on my experience of interaction with subjects, I would prepare a questionnaire as:

Please answer ALL questions for each of the activities.

NA (Not Applicable)

1) Strongly disagree

2) Moderately disagree

3) Slightly disagree

4) Slightly agree

5) Moderately agree

6) Strongly agree

1.My abdominal wall has a huge impact on my health:

$\begin{array}{lllllll}1 & 2 & 3 & 4 & 5 & 6 & \mathrm{NA}\end{array}$

2.My abdominal wall causes me physical pain:

$\begin{array}{lllllll}1 & 2 & 3 & 4 & 5 & 6 & \mathrm{NA}\end{array}$

3.My abdominal wall interferes when I perform strenuous activities eg. Heavy lifting, exercising:

$\begin{array}{lllllll}1 & 2 & 3 & 4 & 5 & 6 & \mathrm{NA}\end{array}$

4.My abdominal wall interferes when I perform moderate activities eg. Bending over, coughing, deep breathing: $1 \quad 2 \quad 3 \quad 4 \quad 56 \quad$ NA

5.My abdominal wall interferes when I walk or climb stairs:

$\begin{array}{lllllll}1 & 2 & 3 & 4 & 5 & 6 & \mathrm{NA}\end{array}$

6.My abdominal wall interferes when I dress myself, take showers or cook(activities of daily living):

$\begin{array}{lllllll}1 & 2 & 3 & 4 & 5 & 6 & \mathrm{NA}\end{array}$

7. I often stay at home because of my abdominal 
wall (my abdominal wall interferes with my social activities like visiting friends, relatives etc.):

\section{$\begin{array}{lllllll}1 & 2 & 3 & 4 & 5 & 6 & \mathrm{NA}\end{array}$}

8. I accomplish less at home or at work because of my abdominal wall:

$\begin{array}{lllllll}1 & 2 & 3 & 4 & 5 & 6 & \mathrm{NA}\end{array}$

9. My abdominal wall affects how I feel everyday:

$\begin{array}{lllllll}1 & 2 & 3 & 4 & 5 & 6 & \mathrm{NA}\end{array}$

10. I often feel nervous because of my abdominal wall:

\section{$\begin{array}{lllllll}1 & 2 & 3 & 4 & 5 & 6 & \mathrm{NA}\end{array}$}

\section{Conclusion}

QOL as a whole improved in our study. After 3months QOL score is better when compared to pre-operative and QOL scores is even better at 6months compared to pre-operative and postoperative $3{ }^{\text {rd }}$ month. Both CCS and HerQLes are appropriate in assessing QOL after ventral hernia repair in our Indian population. More studies with larger samples are needed to validate HerQLes and also to compare CCS and HerQLes. More studies are also needed to evaluate QOL and to standardize QOL scale according in Indian population.

\section{References}

1. Mark A. Malangoni, Michael J. Rosen, Hernias. In: Townsend, Beauchamp, Evers, "Sabiston Textbook of surgery". $19^{\text {th }}$ edition. New York: an imprint of Elsevier; 2012;1093-1095,1114- 1135.

2. Richard H. Tunage, Kathryn A. Richardson, Benjamin D. Li, "Abdominal wall, Umbilicus, Peritoneum, Mesenteries, Omentum". In: Townsend, Beauchamp, Evers, "Sabiston Textbook of surgery". 18 edition. New York: an imprint of Elsevier;2007;1171- 1179.

3. Robert L. Bell, Neal E. Seymour, "Abdominal wall, Omentum, Mesentery and Retroperitoneum". In: Charles Brunicardi F, "Schwartz Manual of Surgery". $8^{\text {th }}$ Edition, New York: McGraw-Hill; 2006;1221-1296.

4. Richard J. Javid, David C. Brooks, "hernias". In: Michael J. Zinner, Stanley W. Ashley. "Maingot's Abdominal Operations" $11^{\text {th }}$ edition, New York: McGraw-Hill; 2007;122-124.

5. Kristian K. Jensen, Nadia A. Henriksen, Henrik Harling. "Standardized measurement of QOL after incisional hernia repair: a systemic review". The American Journal of Surgery 2014;208(3):485-493.

6. C. O. Thomsen, T. L. Brendum, L. N. J0rgensen, et al."QOL after Ventral hernia Repair with endoscopic component separation technique”. Scandinavian Journal of Surgery 2016;105(1):11-16.

7. B Todd Heniford, Amanda L Walters, Amy E Lincourt, Yuri W Novitsky, William W Hope, Kent W Kercher. "Comparison of Generic versus Specific Quality-of-Life Scales for Mesh Hernia Repairs". J Am Coll Surg 2008; 206(4):638-644.

8. Ladurner, R., Chiapponi, C., Linhuber, Q. et al.’Long term outcome and QOL after open incisional hernia repair-light versus heavy weight meshes".BMC Surg. 2011;11:25.

9. Krpata DM, Schmotzer BJ, Flocke S, et al. "Design and initial implementation of HerQLes: a hernia-related quality-of-life survey to assess abdominal wall function".J Am Coll Surg. 2012;215(5):635-642.

10. Hope WW, Lincourt AE, Newcomb WL, Schmelzer TM, Kercher KW, Heniford BT. "Comparing Quality-of-Life Outcomes in Symptomatic Patients Undergoing Laparoscopic or Open Ventral Hernia Repair". Journal of Laparoendoscopic \& Advanced Surgical Techniques. 2008;18(4):567-571.

11. Colavita PD, Tsirline VB, Belyansky I, et al.“ Prospective, long-term comparison of QOL in laparoscopic versus open ventral hernia repair". Ann Surg.2012;256(5):714722 .

12. Christoffersen MW, Rosenberg J, Jorgensen LN, Bytzer P, Bisgaard T. "Health- related QOL scores changes significantly within the 
first three months after hernia mesh repair". World journal of surgery. 2014;38(7):18521859.

13. Klima DA, Tsirline VB, Belyansky I, et al. "QOL following component separation versus standard open ventral hernia repair for large hernias". Surg Innov. 2014;21(2): 147-154.

14. Mussack T, Ladurner R. Vogel T, et al. "Health-related QOL changes after laparoscopic and open incisional hernia repair: a matched pair analysis". Surg Endosc. 2006;20(3):410-413. 